\title{
CRISPR-Cas Genome Editing Tool: Mechanisms of Pathogen Resistance Plants - Review
}

\author{
Karthik Kalidoss \\ karthikkalidoss87@gmail.com
}

\begin{abstract}
Keywords: CRISPR-Cas9-genome editing- sgRNA expression-crop improvement-disease resistance plants
\end{abstract}

\begin{abstract}
In recent years, the CRISPR-Cas system is most familiar and advance genome editing tool in modern biological research. The genome editing tool used in various biological researchers worldwide in past years has witnessed exposure site-directed mutagenesis moderation methods zinc finger nucleases (ZFNs), Meganucleases, CRISPR-Cas9(associated proteins 9) and transcription activator-like effector nucleases (TALENs), CRISPR-Cas gene editing technology to ease design and implement, more flexible and low-cost. Plants are affected by two types of stresses like biotic and abiotic. Abiotic occurs naturally temperature or wind, sunlight depends upon on the environmental conditions. Biotic stress is caused by pathogens of virus, fungi, bacteria, etc. This review to focal point on the present-day advance for plant protection use these gene editing system mechanism of disease resistant plants in past and current trends of research. A short overview of the experimental methodology for Beet Curly Top Virus (BCTV) disease and Magnaporthe oryzae fungus infection cause rice blast disease resistance mechanisms will be discussed. Furthermore, the need developments of this genome editing tool in future.
\end{abstract}

\section{Introduction}

In thousands of years, humans try to engineer life in layout to develop and enrich useful features in various organisms. The technology of CRISPR/cas9 is of the pre-eminent tool of genome editing technique in genetic engineering and biotechnology research field. The innovation of CRISPR/Cas9 technology has made this fantasy come true. In the gene editing method opens a new era for mammals and plants, for both basic scientific research and genetic engineering techniques. It's extremely simple, low cost, and resourceful in many applications with minor moderations. The technique is swiftly evolving and its application is constantly broadening in the following years [1]. Currently, CRISPR-Cas system a new type of site-specific nucleases has arisen and uplift a revolution in the genomics field. The gene editing technology to taken from the acquired immune system in bacteria and archaea. To pinpoint the phages or foreign plasmids the Cas proteins used cut them. This system containing by Cas genes inserted a plasmid and specifically designed CRISPR's. The advantage of the genome editing technology can be cut the organism genes any desired location and specific gene silencing in 2012 [2]. Nowadays, research fascinated this application and principles of the CRISPRCas system. It's split into three major types I, II and III., specifically the type II more than two types i) tracrRNA (Trans-activating RNA), ii) crRNA (CRISPR-RNA) and RNaseIII (RibonucleaseIII) to edit target genes [3]. The clustered regularly interspaced short palindromic repeats (CRISPR) sequences are cleaved and transcribed into CRISPR-RNA (crRNA) with include 20nt guide sequences, target Cas proteins, DNA conquer complementary sequences. The Cas proteins demolish the conquer DNA sequence double -strand trans-activating small RNA (sgRNA), cas9guides RNA polymerase III (crRNA) enzyme to fully-fledged forming duplex-RNA components [4].

The applications used in plants such as crop improvement for the purpose of agriculture. Furthermore, the severe weather condition, growing population rate, lessens agricultural landscape availability; accumulate biotic, abiotic stresses and nutritional traits notable constraints for farming and food production. CRISPR /cas9 genome editing to help the improvement of crop growth and stress tolerance. First-generation gene editing tools are some drawbacks on presents simple design and cloning methods the Associated proteins 9 (Cas9) target multiple sites with used different guide 
RNAs (gRNA). Evidently, the crops involve reducing off-target cleavage and specific target option for the primary CRISPR/cas9 model. For example Sacas9, Nmcas9, and Stcas9 [5].

Present days, different plant species generally used this technology; lots of CRISPR/cas9 vectors are available, depository of public plasmid web URL address Addgene (http://www.addgene.org/crispr/plant/). The improved computing information storage system is a strong platform for genetic engineering researches [6]. Nowadays, the creation of plants with valuable compositional properties and with traits that give out to the resistance of different biotic and abiotic stresses. During the past few years, several new gene editing systems have been developed; these include CRISPR-cas9, Transcription activator-like effector nucleases (TALENs) and zinc finger nucleases (ZFNs) and [7]. The enchanting scrutiny in plant research New breeding techniques (NBT) and developmental biology many different areas the resistance of abiotic and biotic stresses heterogeneous host cell repair mechanism in gene sequences deletions and small insertions via Homologous Recombination (HR) or micro-homology- mediated end- joining (MMEJ) or homologous end-joining (NHEJ). Meganucleases (MNs), Transcription activator-like effector nucleases (TALENs), Zinc finger Nucleases (ZFNs) and CRISPR-associated protein9 (cas9) the four types nucleases used in genome editing) [8] to comparison of ZFN and TALENs the CRISPR/cas9 is much uncomplicated. For example Zinc finger nucleases (ZFNs) an array of Cys2-His2 ZF domains, each finger to binding specific PAMs, it's difficult to select proper target sequences two ZFNs form a dimer to locate a unique 18-24 bp DNA sequence. Owing to off-target risks, modular DNA binding proteins, requirements of context-dependent binding and ZFN, TALEN applications are very limited. The gene edited crops carrying edited DNA for the required trait such as improved crops use in breeding programs. So, plant biotechnologists use these gene editing tools develop of abiotic and biotic stress tolerance crops [1]. In this review, we discuss previous years CRISPR-cas9 based application for crop improvement of particularly disease resistant plants and also describe at how its work and target gene mechanisms in similar year Researches. Furthermore, this review to help thinking, inductive about advantages, disadvantages and future new evolutions in the CRISPR/cas9 application of genome editing in plants.

\section{The CRISPR-CAS9 general mechanism}

An exciting powerful tool for genetic engineering the CRISPR-Cas9 System gene manipulation a broad spectrum of organisms. It's easy of design, accessibility in use and high effectiveness [9].

\section{Genome editing for Cas9}

The Cas9 RNA-guided endonuclease cleaves DNA at sequences that bind to the crRNA of the Cas9 (RNP). It termed proto-spacer Adjacent Motifs or PAMs Cas9 binding to sequences seize viral or plasmid genome the different cas9 proteins from species of bacteria or archaea identify different PAM sites [10]. Multidomain and multifunctional DNA endonuclease is a large (1,368-amino acid) Cas9 (referred to as SpyCas9) from Streptococcus pyogenes. The upstream of dsDNA 3bp clip of sequence PAM through its two marked nuclease domains: the guide RNA sequence complementary (target strand) and RuvC-like nuclease domain for cleaving the DNA strand opposite complementary (non-target strand).It includes the most critical role of the editing system and crRNA maturation, spacer acquisition also [9]. The two components CRISPR -Cas9 system cas9 and sgRNA via cleaves foreign DNA.Cas9 is a DNA endonuclease enzyme that can be obtained from various bacteria, such as, Brevibacillus laterosporus, Staphylococcus aureus, Streptococcus pyogenes, Streptococcus thermophilus but cas9 isolation broadly used for Streptococcus pyogenes [3]. Invitro fusion of cas9crRNA the major development from Streptococcus pyogenes and Streptococcus thermophilus was able as RNA-guided nuclease, with RNA matching seize sequences and a cas 9 protein producing DSB at a particular position in the DNA. After the proficient of this major function, the process integrates crRNA with tracrRNA was completed to form a single guide RNA [11]. The gene editing system set a powerful platform for specific-sequence genome editing, including site-specific sequence mutagenesis and corrections, gene knock-in, gene knock- out [12]. 


\section{CRISPR Cas vs ZFNs \& TALENs genome editing differentiation}

The comprehension mechanism of the CRISPR/cas system guide RNA( gRNA ) molecule based gene editing tool is assessing better than DNA binding domains-nuclease-based gene modification systems. E.g.: ZFNs \&TALENs. The simplicity of its design and its specificity is very low. However, the effectively targeted gene manipulation and protein generated for each DNA target site the disparity of this system only requires the complement of guide RNA (gRNA) sequence-specific target region. The Cas enzyme can be a guided to the site double-strand break introducing of effect modification and directly inserting RNAs, encoding Cas proteins, sgRNA. Besides, the CRISPR-cas system can produce introduced at one-time multiple gene modifications concurrently because of multiple guide RNAs. Furthermore, the guide RNA strands typically target a DNA sequence of 20bp, which is relatively short, compared to the target sequences of ZFNs \& TALENs. Nevertheless, offer multiple options of all three methods to researchers yet these are not without limitations and complications of off-target effects (mutations introduced as non-specific loci), mosaicism (organisms with a mutant allele in only some of their cells), and difficulties of multiple-gene targeting [11].

\section{CRISPR based genome editing in crop improvements}

The 20 crop species adopted CRISPR based genome editing abiotic and biotic stresses including for different traits yield improve and management. An important role of reporter genes playing in abiotic and biotic stress tolerant mechanisms to improve tolerance, major abiotic stresses like salinity and drought [14] the water stress imparted by drought and temperature, severity is the most endemic abiotic stress that limits plant growth and productivity. Plants respond and adapt to these conditions with an array of physiological and biochemical alterations [13]. The pathogenic microorganisms caused biotic stress to comprise severely challenges in the development of diseases resistance crops. Global Declines need for an account of more than $42 \%$ of potential yield loss and contribute to $15 \%$ food production. The CRISPR-Cas system is a couple of several desirable features, including simplicity, effectiveness, high specificity, minimal off-target effects and amenability to multiplexing and thus seems to be extremely promising in plants. The CRISPR-Cas system simplicity, efficiency, high specificity, minimal off-target effects the mutagenesis of various genes can convey importantly intuition into their function of targeting multiple genes level studies and multigenic agronomic traits in crop plants [14].

\section{Stress-related genes functions in plants}

The Magnaporthe oryzae rice crop successfully accepted to increase the resistance of blast disease the ethylene responsive factor OsERF922 gene expression, disease susceptibility gene factor OsSWEET13.In rice crucial infection by Xanthomonas oryzae pv, OsSWEET13 two knock-out mutants and it's target promoter to lead increase the resistance to bacterial blight disease in ( IR24) indica rice. The remarkable role of (OsAnn3) rice annexins gene examined under cold stress was OsAnn3 CRISPR knockouts, to compared wild-type plants the T1 mutant lines found to cold stress decrease under cold treatment [5]. Dicots Arabidopsis (Arabidopsis thaliana), soybean (Glycine max), tomato (Solanum lycopersicum) and the monocots Brachypodium distachyon, rice (Oryza sativa), sorghum (Sorghum bicolor) and maize genome sizes of the eight species ranged from 120 to $2065 \mathrm{MB}$ which is typical of most land plants, and the GC content ranged from $34 \%$ to $47 \%$. To study the online database CRISPRPLANT (http://www.genome.arizona.edu/crispr/) [15], this technology to develop new crops with improved stress- resistance like a virus- resistant cucumber and climate - tolerant tomato the development of disease resistance in many crops without the need for vast genetic manipulation and backcrossing with the wild source of resistant new technology stimulate and improve [16].

\section{CRISPR-CAS genome editing bioinformatics tools}

Crispr-cas genome editing tools to use in gene editing for other organisms. The bioinformatics included in the collection of unique genes on various organisms. Currently, these databases carry 32 model and other organisms unique genes total of 4680entries and of 223. An enclosed information 
about the organisms, target gene, gene, gene sequences, modifications length, genetic modification, genome editing efficiency, assay, cell line etc., the open source LAMP(Linux Apache MYSQL PHP) server its user- friendly browsing, searching facility is united for easy date retrieval. The present useful tools like BLASTCrisprGE, BLASTNTdb and CRISPR Mapper contemplated potential utilities of CRISPR in the vast area of biology and therapeutics. Analysis tools like BLASTNTdb, BLASTCrisprGE and CRISPR Mapper are also. UniProt, KEGG and Pub Med are other resources to hyperlinked are individual entries [17].

\section{CRISPR/CAS9 genome editing on disease resistant plants}

We are discussing on the disease resistant mechanisms in crops based on CRISPR-Cas gene editing technology. It's how to resist the plants like virus, fungi and bacterial infections. How to transfer the gene in host plants? Specifically, the previous year's gene transfer methodology in the system of CRISPR-Cas. Scientists have achieved incompatible gene silencing technology to produce results using to geminivirus transgenic plants resistant. The site-specific placing of transgenic DNA through the genome editing systems to exploit the HDR pathway. It offers advantages over random insertion methods commonly used in plants (Agrobacterium-mediated transformation and microprojectile bombardment). Now available for non-transgenic and transgenic plants DNA, RNA viruses resistance converse to using CRISPR-Cas technology the systems engineered in plants to target Geminiviruses have consistently reduced virus amassment providing increased resistance to virus infection [18]. So, we can see the CRISPR-Cas induced virus resistance in plants.

\section{Virus Resistance plants}

The CRISPR-Cas9 system of genome editing has been used as a tool for relaying resistance to viruses in plants. It's the approach for protection to plants against Geminiviruses. This system conferred amplified resistance to the plants against the Geminivirus species including $B C T V$-(Beet Curly Top Virus), TMV (Turnip Mosaic Virus) and TYLCV (Tomato Yellow Leaf Curl Virus) [19] advances in genome editing tools used new ways to achieve the improvement of resistance in crops. It's genome editing system to respond to several agricultural challenges, including the acquirement of improved biotic stress resistance. The application of CRISPR/cas tools has been against virus infection, followed by the endeavour to improve fungal and bacterial disease resistance [8]. We discuss the mechanisms of virus resistance genome editing in plants used in CRISPR/cas9.

\section{Genome nature of virus}

Most studies assuming CRISPR-edited plants for virus resistance have targeted ssDNA geminivirus genomes. The virus genome is replicated through a Rolling-Circle Amplification mechanism via dsDNA replicative form or recombination-mediated replication [8]genome nature, they are classified into major groups: double-stranded DNA (dsDNA) viruses with no plant viruses in this group, i) single-stranded DNA (ssDNA), ii) reverse-transcribing viruses, iii) negative-sense single-stranded RNA(ssRNA-),iv)double-stranded RNA, V) positive-sense single-stranded RNA(ssRNA+). Geminiviruses have either one or two circular ssDNA components of, 2.6-2.8 KB encapsulated within twinned quasi-icosahedral particles [20].

\section{Beet curly top virus (BCTV) disease resistance mechanism CRISPR-CAS9}

Geminiviruses frightening agriculture and food security, infecting key crop species, especially in tropical and subtropical regions. The CRISPR- CAS9 system has currently been used to converse molecular immunity against several eukaryotic viruses, including plant DNA geminivirus. Recently classified the family Geminiviridae into seven genera: Becurtovirus, Turncurtovirus, Topocuvirus and Mastrevirus, curtovirus, begomovirus and Eragrovirus [21]. The symptoms of curly top disease (CTD) encompass stunted and distorted plant growth, vein swelling, leaf curling and necrosis of hyperplasic phloem. The disease affects over 300 plant species, ornamentals, including crops, and weeds from at least from 44 families. The curly top has been an economically important disease of sugar beet in this country. Since the first report from Iran in 1967[22].A CRISPR-Cas immune system engineered to target Beet curly top virus (BSCTV) in transgenic N.benthamiana impede virus 
accumulation during transient assays and the strategy of agrobacterium-mediated transformation of leaves with expression plasmid vectors of cas9/sgRNA. The transgenic $A$. thaliana and N.benthamiana plants overexpressing the same CRISPR-Cas system were highly resistant to BSCTV infection. BSCTV accumulation in these plants was inhibited and efficiency of reducing virus hoarding was correlated with the expression level of Cas9. [18].

\section{Methodology of Overexpressing SgRNA-Cas9 Mechanism is Highly Resistant to BSCTV Virus Infection}

\section{Experimental vectors construct for CRISPR/Cas9 system}

The five types of vectors to be used in the experiments pHSN401-A7/ B7/C3\%Cas9 (sgRNA alone), pHSN401 (Cas9 alone), pHSN401- sgRNA (A7, B7 and C3), pHSN401-mA7/mB7/mC3 (mutated sgRNA) and pHSN401-A7/B7/C3-dCas9 (dead Cas9), the mock vector pHSN401\%Cas9\%sgRNA (without Cas9 and sgRNA) was used as the control [23].

\section{Target Genes}

This experiment following to target three types of genes as known as CP (Ceruloplasmin protein), REP (replicase) and IR (Immune response gene) [23].

\section{SgRNA target Selections}

Beet severe curling top virus (BSCTV) genome 1.8 copies were used with hosts of Agra inoculation cambia T-DNA carrier transformation of the complete circular virus genome is recombination generate the BSCTV genome is small $(2.9 \mathrm{~KB})$ and encoded only 7 proteins to identify highly effective sgRNA-Cas 9 target sites, chose 43 candidate sites designed and construct with 43 pHSN401vectors 9 . The Cas 9 driven each contain $2 \times 35 \mathrm{~S}$ promoter and one more sgRNAs drive AtU6promoter.All candidate sites within one half three 300(nt) regions in coding or non-coding BSCTV genome sequences [23].

\section{SgRNA Construction for Cas9-Vectors}

The construction of sgRNA-Cas9 vectors to compare with the control vector, 38 of the 43 constructs reduced with viral DNA by over $60 \%, 80$ percentage plasmids are double-stranded DNA of 20 constructs over reduced it. The Cas9 drive may cleave the pCambia-BSCTV plasmid before it generated a single-stranded circular viral genome. The method to develop directly sgRNA-Cas9 could inhibit actively replicating virus. The target sgRNAs A7, B7 and C3 sites which inhibited virus buildup targeting sites in the transient screening assay [23].

\section{Inoculation BSCTV genome in $N$. benthamiana and Arabidopsis plants transgenic}

The A. tumefaciens strain GV3101 and EHA 105 were used in Arabidopsis and N. benthamiana, individually. $N$. benthamiana transgenic plants, the infiltration method was the same with transient assays of EAH105 containing cambia 1300-BSCTV genome with a final $\mathrm{A}_{600} \mathrm{NM}$ of 0.2. Arabidopsis transgenic plants, containing cambia 1300-BSCTV was grown at $28^{\circ} \mathrm{C} \mathrm{ml}$ of LB medium to avoid some infections with added kanamycin and rifampicin the final $\mathrm{A}^{600} \mathrm{~nm}$ 1.5., Centrifugation and suspended in GV3101 were harvested at $2 \mathrm{ml}$ of LB medium [23].

\section{Cas9, sgRNA expression level analysis and RNA Extraction}

The chemical solution of TRIzol use to extract in total RNA and then treated with enzymes of Recombinant DNase I and reverse transcript into CDNA with Superscripts, sgRNA levels, both oligoDT and a sgRNA-specific primer used with Cas9 and sgRNA expression levels measured by qRTPCR.ACTIN7 protein in Arabidopsis and PPR protein (Penta-tri-co-peptide repeat-containing protein) in N. benthamiana used as internal controls. The expression levels of cas 9 and sgRNA in A7-1 (Arabidopsis) and C3-1 (N. benthamiana) were defined to be 1.0[23]. 
Arabidopsis and N. benthamiana plants genomic DNA was isolated with Cetyl trimethyl ammonium bromide (CTAB) buffer. The qPCR assays, internal control of mechanism by PPR protein. Also, southern blots, total DNA $(2 \mu \mathrm{g})$ were separated by Gel electrophoresis for $14 \mathrm{hrs}$ in $0.8 \%$ agarose gel and transferred to a Hybond $\mathrm{N}+$ Membrane and a 227bp fragment is BSCTV genome used to generate $[\alpha-32 \mathrm{P}]$ dCTP-labelled probes by a Prime-a-Gene labelling system. The device of phosphor Imager used detected in DNA accumulation [23].

\section{Fungus resistance plants}

Fungal diseases generate a considerable loss of yield in many crop plants. The natural host defense mechanism is developed in fungal-resistant transgenic plants. [24]. Recent application of techniques in biotechnology and molecular biology to the host-pathogen interactions study to detect various genes. The numerous genes involved in the response of defense from pathogens. Genes that express proteins, peptides or antimicrobial compounds indirectly or indirectly active general plant defense mechanism in plants. Nowadays, develop the techniques of genetic disease- resistance; plant breeding technology [25] fungi are topmost pathogen of all dependent on plants for their carbon and energy source like most other organisms that are not able to photosynthesis. There are about 250,000species of higher plants, but six times as many 1.5 million species of fungi. The pathogens are cause biotic stress to plants have symbiotic or pathogenic interactions [26]. Currently, the CRISPR-Cas9 genome editing tool used for gene characterization in plant pathology. Its strategies have also been used to edit pathogen effector proteins or/ and knockout methodology [27]. We discuss the mechanisms of fungal resistance genome editing in plants used in CRISPR/cas9.

\section{Genome sizes of Fungi}

The comparative analysis of fungal genomes shows fungi are very varying. A genomic differentiation between $68 \%$ of amino acid sequence identity the following species Aspergillus nidulans, Aspergillus fumigatus and Aspergillus oryzae. The Acomycota, Basidiomycota, Oomycota and Mucoromycotina groups encodes for 11129.45, 15431.51, 24173.33, 13306 no. of genes respectively. In average coding gene sequence in fungi to studied 172 fungal species only seven species have genome sizes larger than $100 \mathrm{mb}$ the small size fungi occurred larger genomes, some of the species given by example: The genome size of Cenococcum geophilum (177.57 MB) is the largest and smallest one is Hansenula polymorpha $(8.97 \mathrm{MB})$ studied species. No single species from Glomeromycota Chytridiomycota, Oomycota, Mucoromycotina, Stramenopiles, has genome size larger than $100 \mathrm{MB}$ etc [28]. 


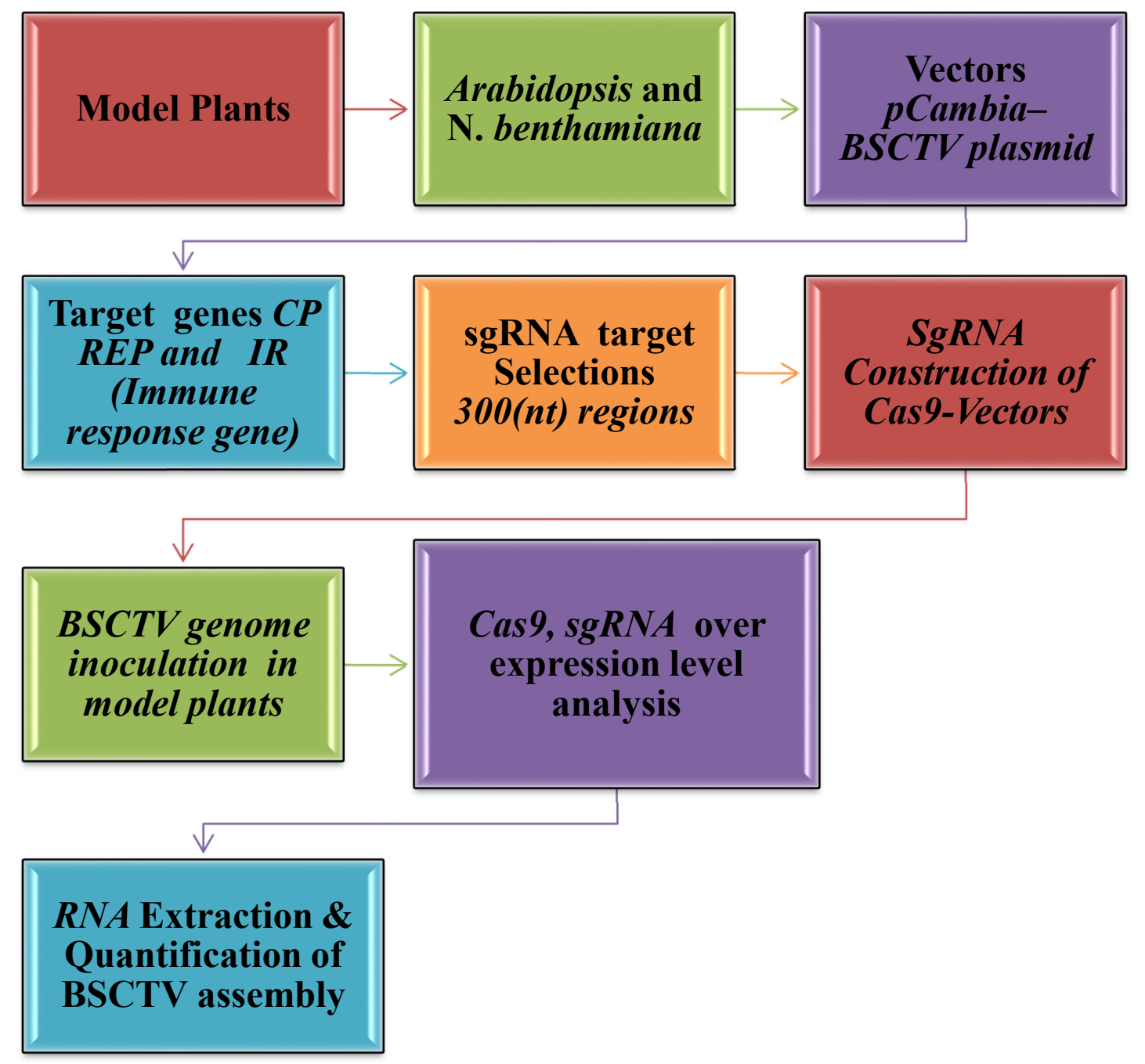

Figure 1. Methodology steps of overexpressing sgRNA-Cas9 is highly resistant to BSCTV virus infection

\section{Rice blast disease resistance mechanism CRISPR-CAS9}

In current years, the CRISPR-associated (cas9) tools for plant genome editing Sequence-Specific Nucleases (SSNs), Transcription Activator-like Effector Nucleases (TALENs) including Zinc-finger Nucleases (ZFNs) the tools used genome editing for particular major crops such as rice, maize, wheat, sorghum, tomato, potatoes and soybeans. Rice blast is one of the most disastrous diseases affecting rice crops in worldwide. In current years, sequence-specific nucleases (SSNs) use gene-specific genome editing tool CRISPR - Cas9 via improvement of crops in rice blast disease resistance engineering by a CRISPR-case 9 SSN (C-ERF922) targeting in the gene of rice OsERF922 [29]. Magnaporthe oryzae is a hemibiotrophic fungal pathogen that causes rice blast disease (Oryza sativa) its infection of rice a germ tube generate from the conidium differentiates into a specialized infectious structure called appressorium, which adheres tightly to the plant surface using mucilage and thin penetration pin riddle the host surface using this pressure to enter a leaf epidermal cell [30]. The infected plants, after a few days of infection biotrophic growth within rice tissues, a necrotrophic like phase associated with the onset of speculation are switched in fungus [31].

We are seeing about the $O S S E C 3 A$ gene expression in various organs and its gene's prospective ability to exocyst complex interact and participated with the several other subunits. In CRISPR -cas9 cause dwarf and a lesion- mimic phenotype to enhanced resistance the fungal pathogen Magnaporthe oryzae defense responses, up-regulated transcript levels of pathogenesis, salicylic acid synthesisrelated genes increased $O \operatorname{ssec} 3 a$ mutant gene mechanisms in the gene editing technology of CRISPR- 
Cas9.In addition, $O S S E C 3 A$ interacted with rice $S N A P 25$-type t-SNARE protein OSSNAP32, which is involved in rice blast resistance [32].

\section{Methodology of Expression, Gene Ossec3a Defense Mechanisms for Magnaporthe Oryzae Fungus Infection \\ Plant Materials}

To study this experiment plant material in Rice (Oryza sativa L. Japonica) and N. benthamiana its need temperature growth condition at $25-28^{\circ} \mathrm{C}$ with $8 \mathrm{hrs}$ darkness and $16 \mathrm{hrs}$ light every day. The rice plants (Oryza sativa L. Japonica) and N. benthamiana roots induced with gene OsSEC3A.The transient expression analysis in $\mathrm{N}$. benthamiana the binary vector pCAMBIA1305-OsSEC3A-GFP was introduced into Agrobacterium strain EHA105, which was used to infiltrate. Its protoplasts were isolated using the same method Arabidopsis plant used to observe with the changes laser scanning confocal microscope [32].

\section{Experimental Vectors constructs for CRISPR/Cas9 system}

The vectors construct in design and method for the CRISPR-Cas9 system in plants. The plasmid construction Gene OSSEC3A cDNA fragment was amplified using 1305OsSEC3AGFP-F/R and cloned into the pCAMBIA1305-GFP vector (produced by insertion of the GFP backbone of pAN580 into the $p C A M B I A 1305$ vector at the SacI-SalI sites) to generate the pCAMBIA1305-D35SOsSEC3A-GFP-NOS vector with single guide RNA(sgRNA). The Cas9 target vector was driven with maize ubiquitin promoter for expression in rice and sgRNA expression, pol III type promoter of U3 sgRNA driven by sgRNA .Binary $T$ - DNA vectors for used were transformation and co-expression of Cas 9 and $\operatorname{sgRNA}[32]$.

\section{Target Genes}

This experiment following to SEC3A (Exocyst complex component) genes from Arabidopsis thaliana plant model organism. The role of exocyst subunit sec3 in plants for root hair elongation, pollen germination and embryogenesis. The exocyst to the plasma membrane EXO70 is name anchored there is no evidence linking SEC3with plant immunity. The ability of geneOsSEC3A to participate in the exocyst complex and obtained OsSEC $3 A$ mutants $(O \operatorname{ssec} 3 a)$ using CRISPR/Cas9 technology [32].

\section{Analysis of gene expression}

$\beta$-glucuronidase (GUS) assay, $2.5 \mathrm{~kb}$ of the OSSEC3A promoter sequence was amplified using OsSEC $3 A$-Pro-F/R, and the resulting construct was transformed into cv. Kitaake by Agrobacterium tumefaciens RNA extraction and transcription-PCRs (qRTPCR-PCRs) assays used OsSEC $3 A$ in plant defense response gene response analyzing via Real-time PCR [32]. 


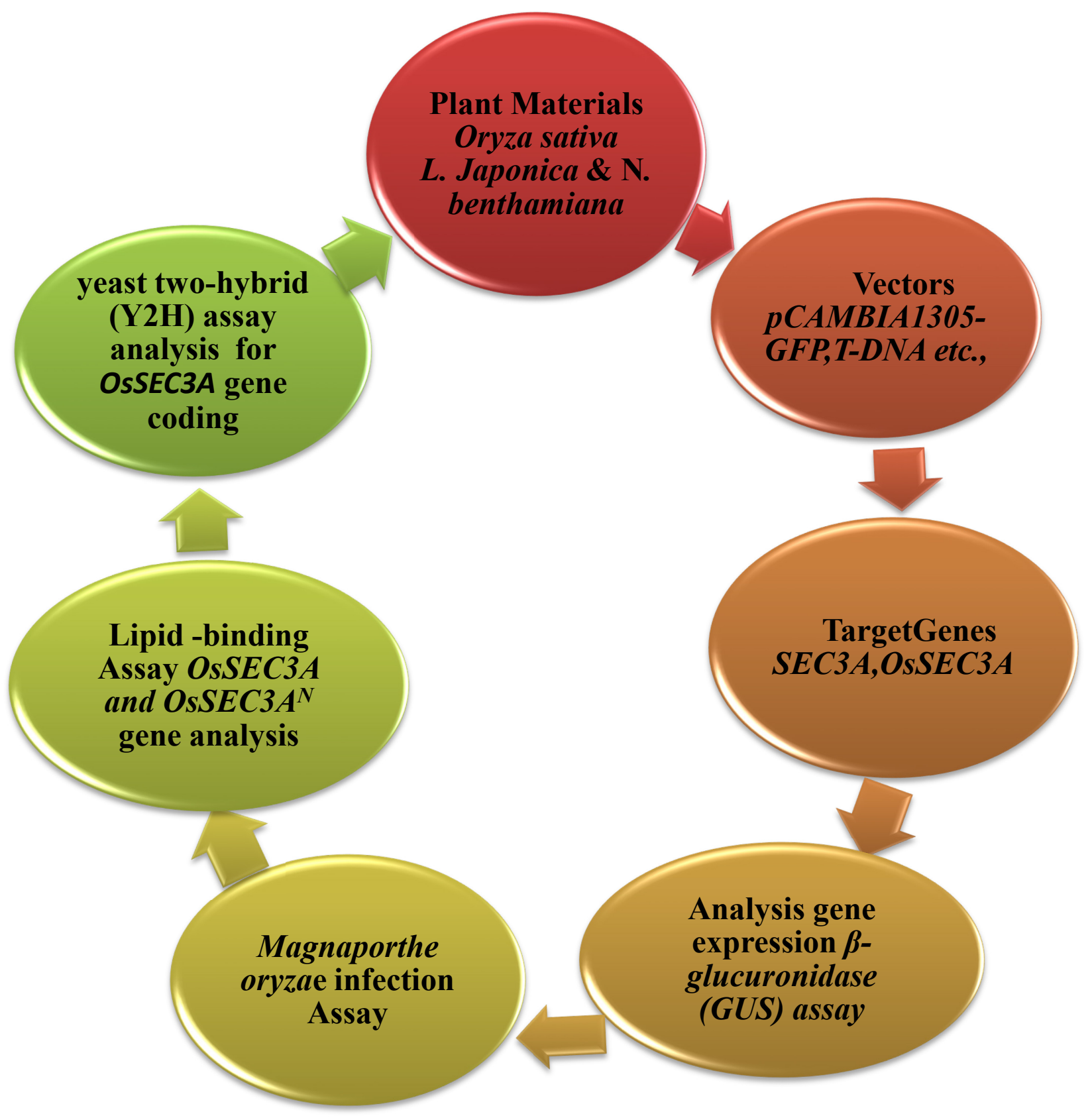

Figure 2. Methodology steps of expression, gene OsSEC3A defense mechanisms for Magnaporthe oryzae fungus infection

\section{Magnaporthe oryzae Infection Assay}

The pathogen Magnaporthe oryzae isolate HLJ07-45-1-2was evaluate for virulence in kitaake and the Ossec $3 a$ mutants. The number and size of lesions in $\sim 35$ inoculated seedlings from each line were evaluated 7 days post-inoculation (dpi). The disease severity the disease symptoms, which reflects the percentage of the leaf area exhibiting necrosis/spots, were analyzed [32]. 


\section{Lipid binding Assay}

The lipid binding assay to analysis the gene expressions of OsSEC 3A and OsSEC $3 \mathrm{~A}^{\mathrm{N}}$ (amino acids 1-450) coding sequences (CDS) were amplified using primers and inserted into vector pMALc2X tagged with MBP using an infusion cloning kit [32].

\section{Interactions of Protein-protein Assays}

The yeast two-hybrid ( $\mathrm{Y} 2 \mathrm{H})$ assay used for analysis, the coding regions of OsSEC $3 A$ and various domain deletion variants of OsSEC3Awere cloned into Y2H bait vector pGBKT7. Saccharomyces cerevisiae strain AH109 used for Bait and prey constructs was co-transformed. The in vitro pulldown assays, the coding regions of target genes OSSEC3A, OsSEC3A $A^{C}$ (amino acids 310-888), OsSNAP32, OsExo70B1 and OsExo70A1 plasmids respectively. The constructs were transiently introduced into N. benthamiana by infiltration with A. tumefaciens strain GV3101. Agrobacteriuminfiltrated leaves were placed into a 96-well microtitre plate with a hole punch and kept in the dark for $5 \mathrm{~min}$. After, to allow detection of luminescence and measured with Dual-Luciferase Reporter Assay System [32].

\section{Future developments of CRISPR-Cas system}

Genome editing tools are assured to have a notable impact on basic and applied research in plant biotechnology. The CRISPR-Cas system genome-editing tool to advantage of editing desired genetic or epigenetic variations. An approach of efficiency for Cas9 and sgRNA specificity and expression can improve [14]. A wonderful genome editing tool of modern biology CRISPR-cas9 to update and develop the research of plant and animal, medical therapeutics etc., the genome editing is the most promising application of CRISPR/Cas9 technology in agriculture. A major challenge is to identify the host proteins and target. The future genome editing approaches studies effectors and characterizes the underlying genes and selects one of many possible targets [8]. The underlying mechanisms of after phage infection target identification, plasmids in transformation, type of target nucleic acid (DNA or RNA) are selected, foreign sequences into CRISPR loci and crRNA binding. The systematic and comprehensive profile of Cas9: a large number of related mutant target sites need to be related in microorganisms, guide RNA-mediated DNA cleavage specificity, generate and measurements. In specifically need to develop of Cas9: guideRNA complexes [11]. CRISPR-Cas9 editing, allowing researchers to make exact edits without knock-ins. The mutation prompts by repair of CRISPR-Cas9 cleavage were non-random and unhesitating by the target sequences. In additionally to develop the more computational tools for the prediction of CRISPR-Cas9 activity [33].

\section{References}

[1] L. Mao et al., CRISPR-Cas9: A powerful tool for crop genome editing- The Crop Journal. (2016) $75-82$.

[2] L.L. Zhang, Q. Zhou, CRISPR/Cas technology: a revolutionary approach for genome engineering. Sci China Life Sci. 57 (2014) 639-640.

[3]Chun et al., Application of CRISPR-Cas9 in Plant biology, Acta Pharmaceutica Sinica B. 7(3) (2017) 292-302.

[4] J. Townson et al., Recent developments in genome editing for potential use in plants, BioscienceHorizons. 10 (2017).

[5] Jaganathan et.al., CRISPR for Crop Improvement: An Update Review, Frontiers in plant Science (2018) Article 985.

[6]Ding et al., RecentAdvancesinGenomeEditingUsingCRISPR/Cas9, Frontiers in Plant Science. 7 (2016) Article 703. 
[7] Ibrokhim et al., Genome Editing in Plants: An Overview of Tools and Applications, International Journal of Agronomy. (2017) 15p.

[8] Borrelli et al., The Enhancement of Plant Disease Resistance Using CRISPR/Cas9Technology, Frontiers in Plant Science. 9 (2018) Article 1245.

[9] F. Jiang, J.A. Doudna, CRISPR-Cas9 Structures and Mechanisms, Annu. Rev. Biophysics. 46 (2017) 505-29.

[10] M. Deborah, T. Schmidt, Te-Wen Lo, Molecular Biology at the Cutting Edge: A Review on CRISPR/CAS9 Gene Editing for Undergraduates. 46(2) (2018) 195-205.

[11] Javed et al., CRISPR-Cas System: History and Prospects as a Genome Editing Tool in Microorganisms, Current Microbiology, Springer Nature, 2018.

[12] Haifeng Wang, Marie La Russa, Lei S. Qi., Recent progress in CRISPR/Cas9 technology, Annu. Rev. Biochem. 85 (2016) 227-264.

[13] Parinita et al., Role of DREB transcription factors in abiotic and biotic stress tolerance in plants, Plant Cell Rep. 25 (2006) 1263-1274.

[14] V. Kumar, M. Jain et al, The CRISPR-Cas system for plant genome editing: advances and opportunities, Journal of Experimental Botany. 66(1) (2015) 47-57.

[15] Luisa Bortesi et al., Patterns of CRISPR/Cas9 activity in plants, animals and microbes, Plant Biotechnology Journal. 14 (2016) 2203-2216.

[16] A.Gal-on et.al, crispr/cas9 genome editing technology is a plant breeding dream comes true, Journal of Plant Pathology. (2016) 98pp.

[17] Karambir Kaur, Himani Tandon, Amit Kumar Gupta, Manoj Kumar et.al .,CrisprGE: a central hub of CRISPR/Cas-based genome editing, Database, (2015), 1-8.

[18] Hu et al., Editing plants for virus resistance using CRISPR-Cas, Acta Vrologica. 61 (2017) 138142.

[19] Khatodia et al., The CRISPR/Cas Genome-Editing Tool: Application in Improvement of Crops, Frontiers of Plant Science. 7 (2016) Article 506.

[20] Mansoor et al., Geminivirus disease complexes: an emerging threat, Trends in Plant Science 8(3) (2003).

[21] Ali et al., CRISPR/Cas9-Mediated Immunity to Gemini viruses: Differential Interference and Evasion, Genome Biology (2015) 16:238

[22] Ameneh et al., Seed Transmission of Beet Curly Top Virus and Beet Curly Top Iran Virus in a Local Cultivar of Petunia in Iran. Viruses. 9 (2017) 299.

[23] Xiang et al, Establishing a CRISPR-Cas-like immune system conferring DNA virus resistance in plants, Nature plants. 1 (2015) 144.

[24] Ignacimuthu et al, Genetic engineering of crop plants for fungal resistance: role of antifungal genes Biotechnol Lett. 34 (2012) 995-1002.

[25] Punja et al, Genetic engineering of plants to enhance resistance to fungal pathogens-a review of progress and future prospects, Can. J. Plant Pathol. 23 (2001) 216-235.

[26] Lattanzio et al., Role of phenolics in the resistance mechanisms of plants against fungal pathogens and insects, Phyto Chemistry: Advances in Research. (2006) 23-67.

[27] Fister et al., Transient Expression of CRISPR/Cas9 Machinery TargetingTcNPR3 Enhances Defense Response in Theobroma cacao, Frontiers in Plants Science. 9 (2018) Article 268. 
[28] Mohanta et al., The diversity of fungal genome- Mohanta and Bae Biomed central, Biological Procedures Online. 17 (2015) 8.

29] Fujun et.al, Enhanced Rice Blast Resistance by CRISPR/Cas9-Targeted Mutagenesis of the ERF Transcription Factor Gene OsERF922.plos ONE11 (4), (2016).

[30] Park et.al. Rice Blast Fungus (Magnaporthe oryzae) Infects Arabidopsis via a Mechanism Distinct from That required for the Infection of Rice ${ }^{1[\mathrm{~W}][\mathrm{OA}]}$ Plant Physiology, (2009), Vol. 149, pp. 474-486.

[31] Ribot et.al, Susceptibility of rice to the blast fungus, Magnaporthe grisea, Journal of Plant physiology (2008)114-124.

[32] Ma et.al, Disruption of OsSEC3A increases the content of salicylic acid and induces plant defense responses in rice, Journal of Experimental Botany. 69(5) (2018) 1051-1064.

[33] O.W. Laurence et al., The Current State and Future of CRISPR-Cas9 gRNA Design Tools, Frontiers in Pharmacology. 9 (2018) Article 749. 